\title{
Renewable Hybrids Grid-Connection Using Converter Interferences
}

\author{
Saeed Jahdi, Loi Lei Lai, Daneil Nankoo \\ Power Systems laboratory; Electrical Engineering Department, City University London
}

\begin{abstract}
Use of Renewable Energy power sources is the best possible solution today to reduce increasingly risk of global warming and the most important type of renewable is Wind and Solar energies which are the most efficient. The green power generation resources are used power generators in Distributed Generation (DGs) sources that are in direct relation with the use of micro capacity power generating units of power system that are installed in distribution level of power systems or all segments that loads and energy consumers are located.

Hybrid systems vary in models. The best hybrid model available today is combination of grid connected wind turbines and solar PV cells that can compensate each other in the grid connected state. In addition, solar cells provide electricity required in day-time while wind turbines compensate the power needed in the night period. Solar cells are consisted of a series of assembly of different cells together to form a flat photovoltaic system to absorb the photons and generate electricity by electrons energized in the circuit. On the other hand, Systems for conversion of energy of wind use PM Synchronous Generators. Recently, wind turbines are even enhanced to use VSD drives to provide the machine the ability of generation in cases that rotational speed varies with changes in speed of wind. The simplified version of the solar-wind hybrid system is provided and Simulations are done to confirm expectation outcomes of this connection.
\end{abstract}

\section{Introduction}

DG resources used in systems by using different types of renewable energy sources available. Today there are different technologies of renewable energy sources and each of them has specific characteristics and positive and negative points. Of them Solar energy, fuel-cell technology and biomass are more into practice. Meanwhile the most important and common source of renewable energy is the wind energy. This is since wind energy has some special advantages in comparison with rest of renewables sources. Use of wind energy is convenient, comes cheap and it's globally accessible in the world. In the mean time there are certain disadvantages for wind systems that should be considered too. The main one is that the wind speed and situation of weather predictions is not easily forecasted and hence presence of energy will face a big question mark. [1]
There are some limitations on the maximum and minimum of the wind speed for generating electricity which is provided by operators and these boundaries should be met to provide a better situation for wind energy generations. Because of this, power systems cannot rely on wind generation solely and there should be back serves for the systems using wind generation to support them in case of calm weather. Often these back up services are provided with another sort of renewable energy source and the main back up for these services can be considered as solar to provide a hybrid system of wind and solar systems. [2]

There are multiple reasons that why this systems are used today. In brief it could be stated that main reasons for increase in use of renewable energy sources is increase of worries about climate change and increase of temperature in the world. Another main reason is increase in energy demands and acceleration of energy consumptions in the world and the final reason is that fossil fuels are forecasted to reach an end in near future and this will make energy providers to look towards new replacements for conventional fossil fuels systems. [8] To provide a comparison between renewable sources and fossil fuel sources, it should be noted that about $1 \mathrm{~kg}$ of $\mathrm{CO}_{2}$ is saved by using each $\mathrm{kW} / \mathrm{h}$ of energy generated by renewables.[7] Energy resources like oil, gas, coal, etc. providing the main sources of today energy in the world and this should be changed in near future using renewable sources. The high level of $\mathrm{CO}_{2}$ emissions can have dangerous effects on weather and environment and can result in changes in environment. For these reasons, renewable sources should be developed further and be used in the world as a replacement source.

\section{Wind Power}

Wind energy is the energy of air motion around the earth surface and it is considered as a mechanical energy source. The power of wind energy nowadays is used to motion power turbines and generate electricity. The level of generated electricity is depended on the level of wind speed and design of the turbines. In this paper, a wind turbine parallel to a solar power panel is used to simulate the power generation of a hybrid renewable system and study the output when connected to power grid. The Wind turbines output power is dependent on speed of wind. 


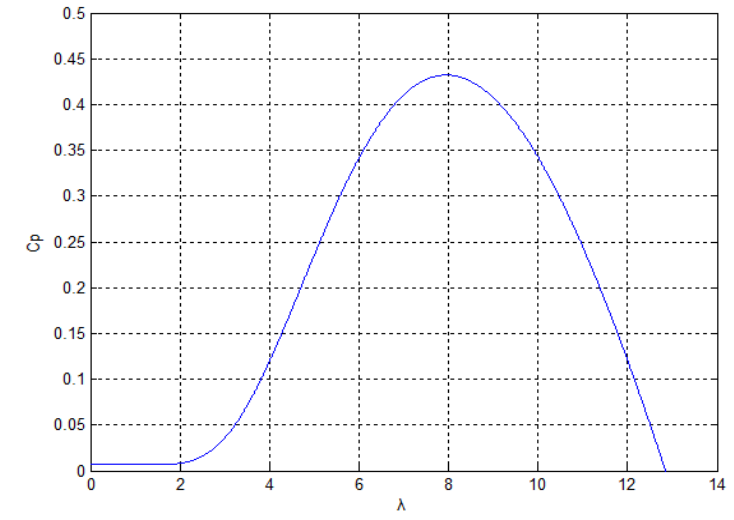

Figure 1. Wind Rotor Power Coefficient Cp on Tip Speed Ratio $\lambda$

\section{Grid Connection Power Electronic equipments}

DC/AC-DC-AC converters are used to solve connection problems of hybrid resources to grid as DG connections. The main concern is differences of wind speed and frequency of system which force us to use some interference equipments to change the frequency and keep it in the adequate boundaries provided by operator. The inverters/converters are used in connection of wind energy systems to grid to facilitate the frequency variations smoother. [3]

Rectifiers and inverters are used in to change the frequencies and enhance amplitude of grid connection voltage as well. Since both voltage and frequency of connection should stay stable, these $\mathrm{AC}-\mathrm{DC}-\mathrm{AC}$ connections in wind turbines and $\mathrm{DC}$ DC-AC connections in photovoltaic cells are used. [4] These converters used include a few circuits for rectifiers, inverters in $\mathrm{AC}$ sides and harmonic and ripple filters that are usually of type of LCs. So the output voltages are kept constant and provided by outcome of rectifiers, the filters in the DC section eliminate harmonics and provide a smoother outcome and the voltage levels and waveform states are controlled in the inverter parts of the system. The DC voltage is provided by the connection section of converter and a PWM inverter can be used to provide an AC voltage as output from the input DC voltages. PWM inverters have good performance in both steady state and transient parts of the system. [6]

The important point on connection of wind turbines in systems is that phase, amplitude of voltage, frequency and many other factors should meet the system specifications that wind or solar system wants to be connected to as hybrid resources. These parameters are determined and changed by using power electronic equipments used in system, hence the role of these devices become more important. [7] AC inverters have the duty of changing the DC power (including both current and voltage) to $\mathrm{AC}$ power which can be used in purposes of real usages in domestic or industrial section. DC converters have the duty of changing amplitude of voltage in photovoltaic (Solar) systems and hence it acts as transformer but for DC sources. The rectifier in the system plays an extra- ordinary role to make the voltage of output stable and consequently cause it to be ready to be used as inverter inputs.

All these in photovoltaic systems matter since the appliances are using alternative current sources and active power generated by solar cells are of direct current type. In addition, even for wind turbines which can provide an alternative current the changes and variations in frequency should be monitored in details and in cases of any frequency deviation, the AC-DC-AC converter system should come into effect. [10] Schematic of the connection of hybrid is simulated in MATLAB and is visible in below to show the inverter and the connection necessary for wind and solar.

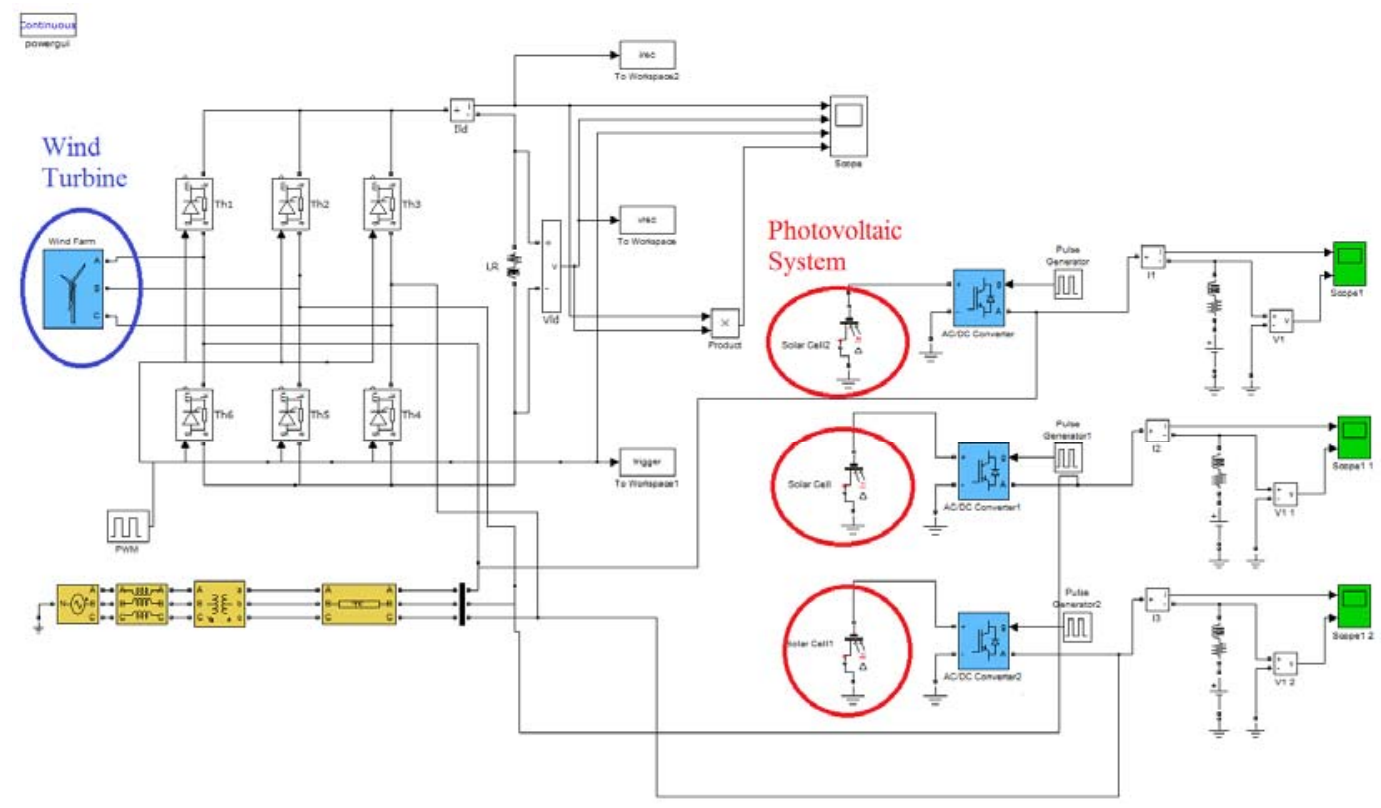

Figure.2. Shematic of Connection usuing Inverters/Converters 


\section{PV Solar Considerations}

Solar systems are used in various applications since there are no fuel cost associated with their fuels, they can work without special supervision and they are relatively reliable. They are getting economical since other fuel sources are getting more expensive every day and they use of them is more risky in future. The solar cells can be used in housings, farms, water pumping equipment and portable telecommunication devices such as satellite or navigators. The case of using PV solar panels should be compared with conventional sources of fuel to see whether it is still economical or not.

They can be used in PV power stations or the distribution section of power systems. The important point about PV power panels is that the energy it is providing in the day is relatively higher than what can be provided in the night when there is no photons striking the $P V$ panel and since the energy cannot be saved in batteries, there should be a compensation method for the level of the power essential in the day. The best possible compensator for this can be the use of another renewable source such as wind turbines.

\section{Renewable Hybrids as DG Sources in Power connection Grid}

Hybrid DGs are produced by connections of wind and photovoltaic sources to provide a more stable power source. These Solar cells are getting very efficient everyday and have the ability to generate a relatively high amount of energy and some parts of this energy is saved in batteries and UPS units to be used in darkness situations or in cases there is no wind blowings. On the other hand, Wind energy is of most environmental friendly energy sources available and this energy is provided by rotation of wind turbines and generation of the necessary power. In below you may see the schematic for the connection of wind and solar photovoltaic cells together to form necessary system of connection. The interconnection of solar and wind in the grid omits the necessity of having extensive UPS units.

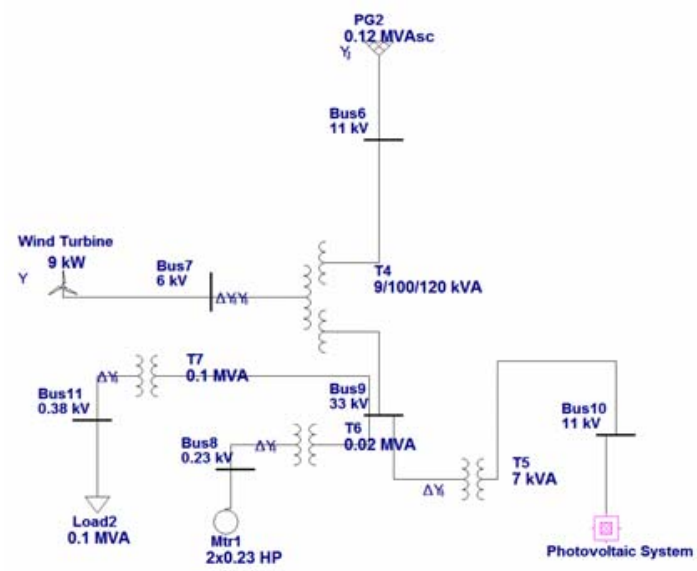

Figure 3. Simulation of Connection in ETAP

\section{Power supply}

Electricity companies normally guarantee a supply peak under a normal set of operating conditions of the network. In other words to supply power during peak hours, in countries where multi rating system applies is a clear advantage for the user.

\section{Emergency power}

Under the circumstances when a part of the supply network may face technical problem, DG may be used to supply power in an emergency. This would obviously reduce the number of people who might have to face electrical black out (power outage).

\section{Even load distribution}

Use of DG is known to improve load distribution in the network. Engineering research shows that DG units tend to change the direction of the current and thus help improve load distribution.

Improvement in the quality of power and reliability

DG is said to improve power quality and offers higher reliability. This according to IEA is the most important feature of such electric power generators. Where DG's are directly connected to the subscriber, should there be any interruptions or cut-off in the distribution network, the subscriber's electricity could be ensured through the use of islanding.

\section{Improved voltage profile}

In connecting DG to the distribution network, if efforts are undertaken to optimize positioning, this would enhance voltage profile. This is particularly important with feeders of considerable length where voltage drops are sensitive.

\section{Improved service life of equipments}

One of the virtues (advantages) of using DG is the reduction in peak loads it offers. All parts and components used in electricity industries have on them some limits or other such as thermal limit, allowable range of current or voltage and alike. In other words, functioning of such parts and components in over Load conditions obviously makes them more vulnerable to damage, fatigue and Shorter service lives. It is clear therefore that use of DG helps greatly in these Respects.

\section{Reduction in losses}

Use of DG in distributed posts results in reduced current flow in lines used between big generators and distribution transformers. Since losses in lines and other elements used in the network vary as the square of current therefore Losses are reduced through load compensation undertaken by DG. This effect is particularly evident during peak loads. This reduction in losses brings with it other benefits related to secondary services and network expenses.

\section{DG and environment}

According to the Kyoto protocol, member countries of the European Union are obligated to fundamental reductions in green house gases. It $\mathrm{s}$ believed that in the united kingdom alone, something like $45 \%$ of carbon pollution by the end of the first 
decade of this century is due to production of electric power. The UK Is therefore determined to obtain $10 \%$ of its total electricity production by 2010 Through the use of renewable energy sources. It is hoped that by the end of the Next decade (2020) the U.K is able to double this percentage, thereby reducing Carbon pollution (resulting from conventional methods) considerably. This Acquisition of electricity is planned to be through the use of renewable sources like solar and biomass. [8]

Problem associated with connecting DG to distribution network

Connecting DG to distribution network, despite all its virtues, has some Disadvantages also. In such case, DG causes harmonics in the network and Reduces short circuit impedance. Also during failure of the network and blackouts if DG is functioning as islanding, then repairmen face obvious dangers. Within the network with the current traversing back and forth; can cause Voltage variation which can increase appreciably as opposed to the case where Current was flowing in one direction only high to low. That local burst between the active and passive power would very quickly divert the voltage. One Way of improving the power quality and disposing of the harmonics present in Current and voltage waveforms present in the distribution network, is the use Of filters. Different arrangements of active and reactive filters a hybrid type, is much more effective. Use of hybrid type filters allows us to make the most of both worlds, so to speak while avoiding the cons of each when used separately.

DG is usually connected to systems by using energy sources to provide a better energy security level and mitigate the risk of climate change that is occurred due to increase in use of fossil fuels. As it is seen in below network, a simple standard IEEE power system including six buses is provided to do a simple simulation over DG impact on power systems. It is seen that presence of DG sources in network causes lower system power loss and improved voltage profile. DG sources should be connected to system in a manner that makes it possible to show flexibility with main generators. The DGs are usually considered as renewables.

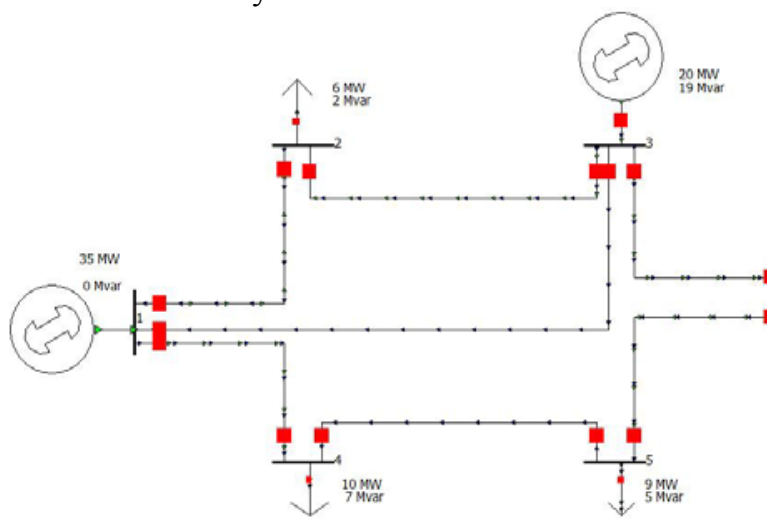

Figure 4. Network With Presence of DG resources

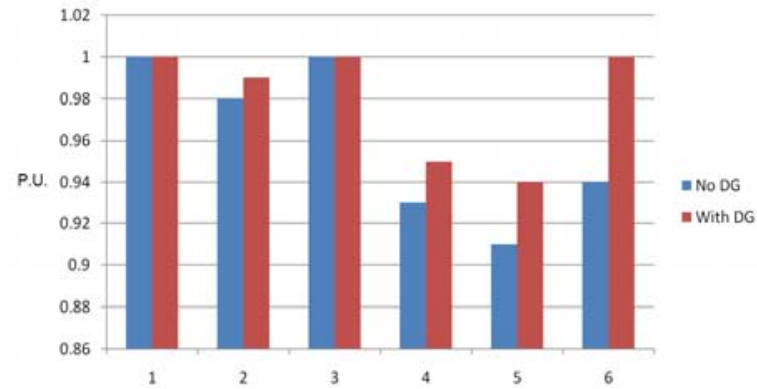

Figure 5. voltage profile in presence and nonpresence of DGs

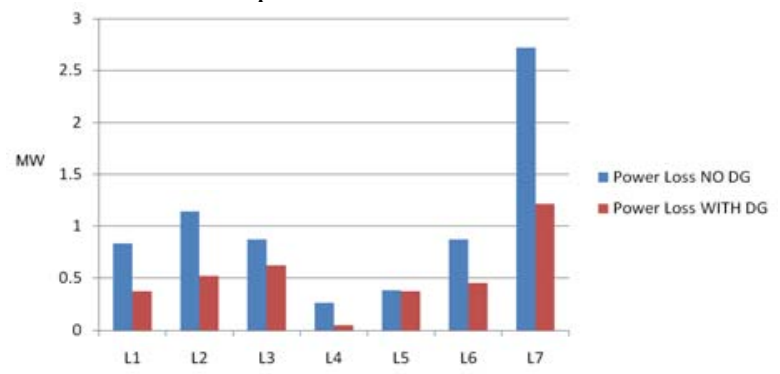

Figure 6. Power loss comparison by considering presence of DG

It is observed that with presence of DGs in the network, there are considerable improvements seen in the network and voltage profile levels while lines' power losses in the network are decreased. Results confirm that DGs presence in network will obviously result in reductions in power losses of system. Presence of DGs in the gird connected network is more highlighted when it comes to its effect on network power and voltage profile levels. Presences of DG units also increase efficiency of system. Other benefits are Reduction in number of overloaded feeders and improved transmission state.

\section{Simulation Results}

A $11 \mathrm{kV}$ and $16 \mathrm{~kW}$ system which is a connection of a $9 \mathrm{~kW} 15$-metter tall wind tower and a $7 \mathrm{~kW}$ solar system is used for simulations. This hybrid system is consisting of a wind generator connected with $\mathrm{AC}$ inverter to system with a DC photovoltaic system connected with a converter and an inverter to system. Relative reactive compensations are designed inside each unit as a form of a shunt capacitor with main power generation system providing active power for the system. Filters are installed in the AC-DC-AC converters to eliminate the ripples. The main duty of this system is regulating voltage and to provide a suitable $\mathrm{AC}$ voltage. A 3 phase inductance and capacitance are used as an AC filter and are applied to eliminate the harmonics. Several transformers have the duty of changing the voltage levels between buses. Simulation results are shown below. The photovoltaic system consists of 42 cells each 
generating 170 watts and charging a battery of 24 volts with a power of 840 Amps.

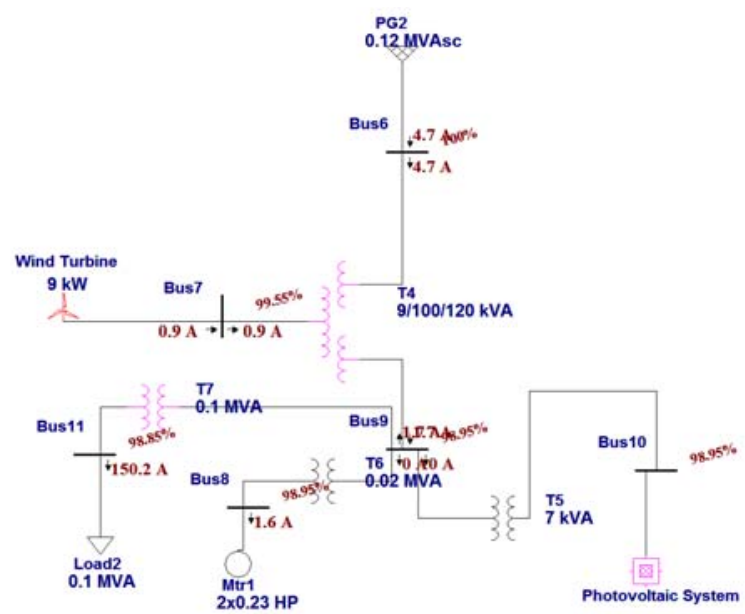

Figure 7. Schematic of grid connection simulated in

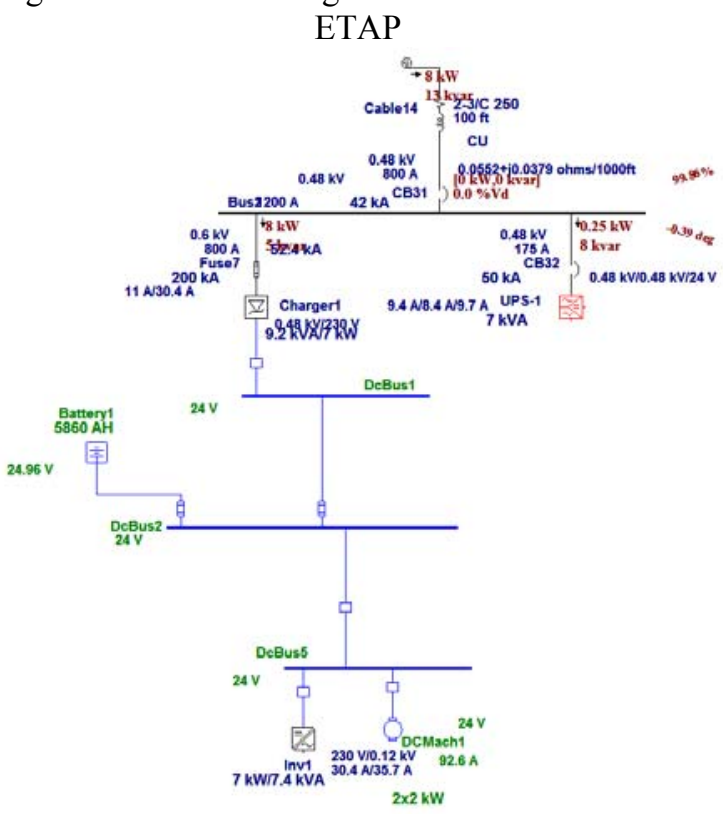

Figure 8. DC Schematic connection of Solar Photovoltaic systems

Simulation results of this system state that the gird connection of Solar system and wind generation can improve the voltage level and power transfer that were lacking due to unpredictability of wind's speed and non-presence of sun and consequently improve voltage and stability of power flow in the network. Final output power is more reliable due to double feeds in the system (both Solar cells and Wind Turbines). In the hybrid systems in DGs technology, grid connection will improve the security of supply and will enhance the financial aspects of the renewable usages in system. A $11 \mathrm{KV}$ power system is provided to simulate the network and results which are visible in Figure 9 show that connection of solar system and wind as hybrid DG sources can improve the power quality and voltages of the DGs.
Usually DG sources can be considered as beneficial from the point of economics especially in places with distances from main grid, they can act as second row generation units and can provide safer supply systems for hospitals and similar facilities requiring safe support of electricity. They can enhance the condition of quality parameters in power and can provide the first level of power generation after a sudden power outage happening. Among the quality of power parameters, the less losses, enhance stability and reliability conditions, decreasing the level of grid congestion and reactive compensation required and definitely significant reductions in costs can be mentioned. And, In DG generators are close to the load distribution level and hence transmission costs are reduced. Mobility of units and nonsensitiveness of system of outgo of one DG unit can be considered as advantages of these sources too.

The main system is provided in figure.7. As it is seen there are one resistive load and one motor load connected to system, the main grid is supporting the system by 0.12 MVAsc and the photovoltaic and wind units are connected to system via proper transformers. As it is seen form Solar system simulated in Figure. 8 the electricity generated in $P V$ panels is in DC form and is used for two purposes. The main portion of this energy provided is injected into power system via proper interferences while the rest of it is used to feed the two 24 Volts DC motors each using $2 \mathrm{~kW}$ of energy. The inverter for DC-AC connection has a capacity of $7.4 \mathrm{kVA}$ out of which 7 $\mathrm{kW}$ is active power. A battery is also connected to DC Bus 2 which can reserve energy for up to 5860 $\mathrm{AH}$ and its actual level of voltage is considered as 244.96 Volts. Also a UPS unit with capacity of 7 $\mathrm{kVA}$ is connected to system to reserve energy as well. The total power expected to be generated by solar power is about $7 \mathrm{~kW}$ too, so the power can be nearly fully saved in UPS and batteries. Transformers are considered as Delta-Star (earthed) transformer models to exclude harmonics from the system by circulating them in transformers' themselves to prevent current ripples.

As the results of Load Flow by ETAP provides in figure.9, it is seen that voltage angles of system is stayed stable and the sequence of -30 (a), -150 (b) and 90 (c) has kept its homogeny in the system and loads are perfectly feeded. The voltage drop in photovoltaic side is only about $1.05 \%$ while in wind connected bus it is even more improved to only $0.45 \%$ which shows an incredible enhancement in better condition for voltage levels after hybrid connection to support main grid's generators and improve feeding conditions quality.

Generally, the case is showing that voltage and currents of feeding the loads has regulated to a better format and this means enhancements in power quality parameters of hybrid systems in comparison with the same system with no renewable connection. 


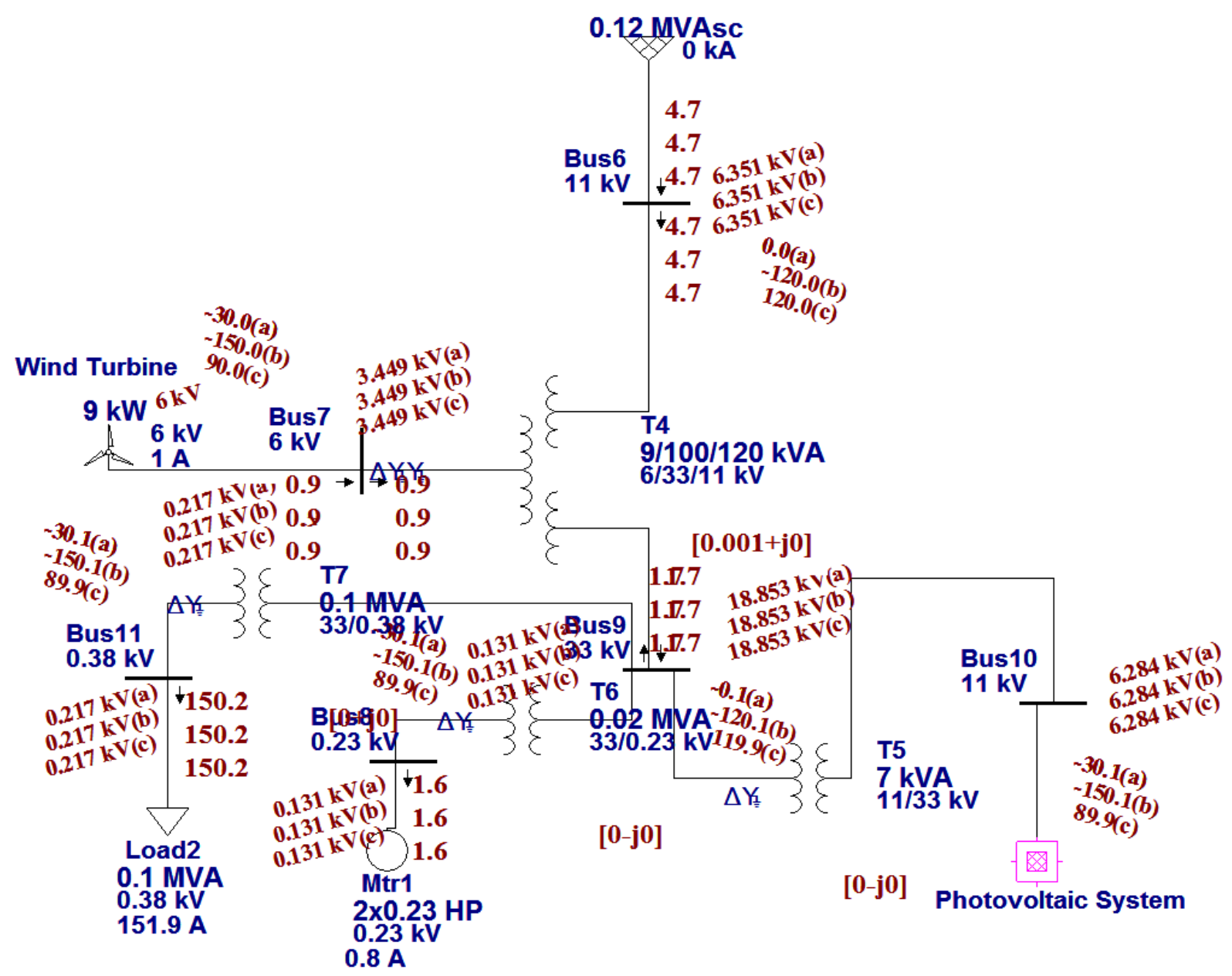

Fig. 9. Connection of DC/AC systems to Grid

The converters in DC section have enhanced the voltage level to be delivered to inverters to be inverted to $\mathrm{AC}$ condition while the wind system has been assumed to act as a synchronous generator in synchronism with system while in practical cases, the voltage of wind turbines should pass through proper inverters and converters too. The protection equipments have not been considered in this simulation but there should be both normal protection predictions and also the protections and detections against islanding operation of both renewable units and also the reverse power flows.

\section{Conclusion}

In this paper, connection considerations of wind and Solar hybrid systems that are connected to grid are discussed and simulations are done in ETAP. Wind turbines are presented as changeable source by time and solar systems are simulated as DC systems connected to grid using DC-AC Inverters in ETAP.
In Hybrid systems of Solar and wind, the capacity is compensated by each other in different cases. Simulation results show that such equipments can improve the situation of power delivered to system. However, it could be a problem if there was no wind in the night. Facilities are affordable and domestic users can provide themselves a micro generation system in their own homes and accordingly reduce natural gas consumption by renewable energy sources as sort of replacement energy.

\section{References}

[1] Jian ; Che Yanbo ; Zhao Lihua ; "Design and research of offgrid wind-solar hybrid power generation systems" , Power Electronics Systems and Applications (PESA), 2011 4th International Conference on Date : 8-10 June 2011, page(s): $1-5$

[2] Yuehua Huang ; Yang Xu ; Xingchen Zhou ; "Study on wind-solar hybrid generating system control strategy", Multimedia Technology (ICMT), International Conference on, 26-28 July 2011, pages: 773 - 776 
[3] Fengge Zhang ; Yuxin Wang ; Erxin Shang ; "Design and Realization of Controller in Wind Solar Hybrid Generating System", 12-15 Oct. 2008

[4] Mao Meiqin ; Su Jianhui ; Liuchen Chang ; Zhang Guorong ; Zhou Yuzhu ; "Controller for $1 \mathrm{~kW}-5 \mathrm{~kW}$ wind-solar hybrid generation systems", Electrical and Computer Engineering, 2008. CCECE 2008. Canadian Conference on, Date: 4-7 May 2008, pages: $001175-001178$

[5] Georges, S. ; Slaoui, F.H. ; "Case Study of Hybrid WindSolar Power Systems for Street Lighting", Systems Engineering (ICSEng), 2011 21st International Conference on, Date: 16-18 Aug. 2011, pages: $82-85$

[6] Reddy, J.B. ; Reddy, D.N. ; "Probabilistic performance assessment of a roof top wind, solar photo voltaic hybrid energy system", Reliability and Maintainability, 2004 Annual Symposium - RAMS, Date : 26-29 Jan. 2004, pages: $654-658$

[7] Min Li ; Jie Wu ; Jun Zeng ; La-Mei Gao ; "Power dispatching of distributed wind-Solar power generation hybrid system based on genetic algorithm", Power Electronics Systems and Applications, 2009. PESA 2009. 3rd International Conference on, Date : 20-22 May 2009

[8] Gupta, S.C. ; Kumar, Y. ; Agnihotri, Gayatri ; "Optimal sizing of solar-wind hybrid system", Information and Communication Technology in Electrical Sciences (ICTES 2007), 2007. ICTES. IET-UK International Conference on, Date : 20-22 Dec. 2007, pages: $282-287$

[9] Ambia, M.N. ; Islam, M.K. ; Shoeb, M.A. ; Maruf, M.N.I. ; Mohsin, A.S.M. ; "An analysis \& design on micro generation of a domestic solar-wind hybrid energy system for rural \& remote areas - perspective Bangladesh", Mechanical and Electronics Engineering (ICMEE), 2nd International Conference on, 1-3 Aug 2010, page: V2-107 V2-110

[10] Fesli, U. ; Bayir, R. ; Ozer, M. ; "Design and implementation of a domestic solar-wind hybrid energy system", Electrical and Electronics Engineering 2009. ELECO'09, Date: 5-8 Nov. 2009, pages: I-29 - I-33 